\title{
Spontaneous spinal epidural hematoma in two toddlers: diagnostic pitfalls
}

\author{
Nathalie Smeets ${ }^{1}$, Machiel van den Akker ${ }^{2}$, Bram Peters ${ }^{3}$, Tim Vanderhasselt ${ }^{4}$ and Anna Jansen ${ }^{1}$ \\ ${ }^{1}$ Department of Pediatrics, Pediatric Neurology Unit, University Hospital Brussels, Belgium \\ ${ }^{2}$ Department of Pediatrics, Pediatric Hematology, Queen Paola's Children's Hospital, Belgium \\ ${ }^{3}$ Department of Pediatrics, University Hospital Brussels, Belgium \\ ${ }^{4}$ Department of Radiology, University Hospital Brussels, Belgium
}

\begin{abstract}
We report two boys with a spontaneous spinal epidural hematoma (SSEH). The first child presented at the age of 2 years with back pain and general weakness after a respiratory tract infection. After a first surgical intervention he developed a recurrence of bleeding, necessitating re-intervention. He developed medullary ischemia. Important neurologic deficits with paresis of the lower extremities and bladder dysfunction persisted. The other child presented at the age of 1,5 years with irritability and crying, attributed to a mesenteric lymphadenitis. He underwent a laminectomy with drainage of the hematoma. The postoperative course was uneventful. In both cases, no identifiable cause was found for the bleeding.

SSEH is a rare condition, but represents a medical emergency. In most pediatric cases, presentation is atypical with back or neck pain and general irritability as sole initial symptom. Neurologic focal signs can arise hours or days after the onset of the bleeding. Knowledge of SSEH presenting signs and symptoms is important for timely diagnosis and treatment. Although it is mostly a solitary event, we stress the importance of close clinical, and if necessary radiological, follow up.
\end{abstract}

\section{Introduction}

Spontaneous spinal epidural hematoma (SSEH) is a rare condition. The incidence is estimated at 0,1 per 100,000 per year in the general population [1].

In the literature, SSEH is defined either as a spinal epidural hematoma ( $\mathrm{SEH}$ ) occurring in the absence of trauma or iatrogenic procedure, or as a true idiopathic SEH without predisposing factors. Predisposing factors include coagulopathy, vascular malformations, tumors, anticoagulant therapy or infections [2,3]. A change in intraabdominal and intrathoracic pressure by, for example, coughing and weight lifting, in combination with the lack of valves in the epidural venous plexus, has been described as a possible explanation for the vulnerability to bleeding in this area [2-4].

A case review by Kreppel [3] describes idiopathic SSEH in one third of all cases.

Symptoms arise from spinal cord compression due to extravasation of blood into the epidural spaces. Presentation in children can be unclear and atypical $[2,4,5]$.

We report a 1.5 -year-old boy with SSEH and full recovery after surgery as well as a 2-year-old boy with SSEH who developed a second hematoma within 3 days after initial surgical decompression. To our knowledge recurrent SSEH is rare. We compare our cases with the literature and discuss possible mechanisms of recurrence.

\section{Case report 1}

A 2-year-old boy presented at the emergency department with fever, coughing, back pain and general weakness with loss of appetite. He was treated with oral amoxicillin for bilateral otitis since three days, with no effect on fever and clinical symptoms. On physical examination, he was pyretic and pale. He had a bilateral otitis and there were reinforced breath sounds at the right side of the chest. Physical neurologic examination at that moment did not show any obvious deficits. Laboratory examination at admission showed a CRP of $<0,5 \mathrm{mg} / \mathrm{l}$ and a white blood cell count of $10.000 / \mathrm{mm}^{3}$. Chest $\mathrm{X}$-ray revealed pneumonia of the right middle lobe and treatment with intravenous amoxicillin was installed. There was a fast resolution of fever, but the back pain worsened. One day after admission, he developed an ataxic gait pattern. Tendon reflexes were normal. Level of consciousness was normal. The back pain increased, with progressive weakness of trunk and legs until, at day 3 of admission, he refused to walk. At that moment, there was also diminished grip strength of his left hand. Neck extension was impossible and painful. Lumbar puncture, carried out at day 2 after admission showed 33 red blood cells $/ \mathrm{mm}^{3}$ and 20 with blood cells $/ \mathrm{mm}^{3}$ with an elevated protein level of 2050 $\mathrm{mg} / \mathrm{l}$ and normal lactate and glucose levels. Cultures and serology were taken and were negative. A brain and spinal cord MRI was performed three days after admission. It showed an epidural hematoma extending from C5 to T8 with significant spinal cord compression (Figure 1a). There were no intracranial abnormalities. Urgent laminectomy was performed without complications. There was a slow resolution of pain

Correspondence to: Nathalie Smeets, Department of Pediatrics, Pediatric Neurology Unit University Hospital Brussels, Laarbeeklaan 101, 1090 Jette, Belgium, E-mail: nathalie.smeets@uzbrussel.be

Key words: spontaneous, cervical, spinal, epidural hematoma, child, recurrent

Received: October 25, 2016; Accepted: November 14, 2016; Published: November 17, 2016 


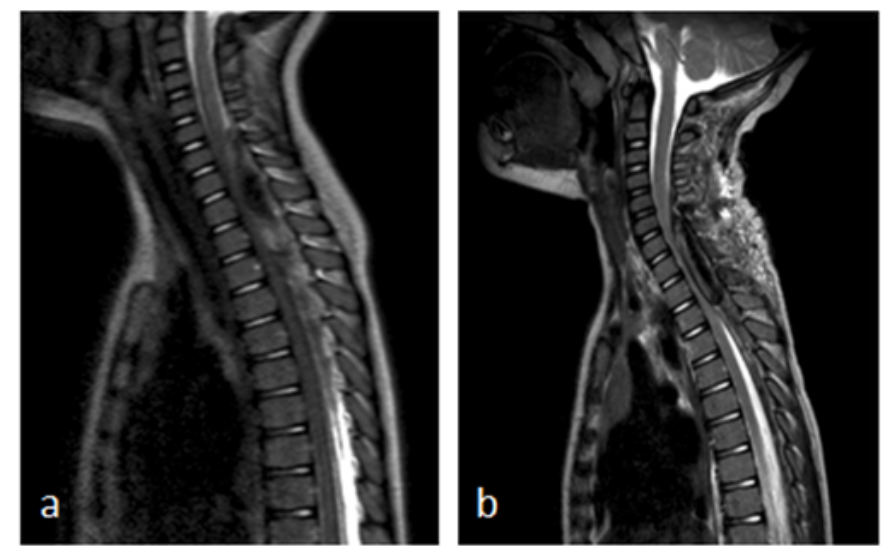

Figure 1. a) Sagittal T2 MRI images show cervical spinal epidural hematoma extending from level C5 to T8 with significant spinal cord compression; b) sagittal T2 MRI images show recurrence of the epidural hematoma, extending from level C6 to T3 with significant spinal cord compression.

and he regained strength in his legs and trunk. However, the left hand function remained impaired. Three days after surgical decompression of the hematoma, there was an acute clinical deterioration with back pain and refusal to walk. Immediate MRI was performed and showed recurrence of the spinal hematoma extending from level C6 to T3 with compression of the cervical dorsal spinal cord (Figure 1b). No underlying pathology (i.e., tumor, vessel malformation) was found on MRI. An evacuation of the hematoma was performed. After surgery, there was a paresis with absent tendon reflexes of the lower limbs and urinary retention. He also developed a miosis and anhidrosis of the right side of the face. Spinal MRI at that moment (three days after re-intervention) showed a central myelopathy with intramedullary edema from C5 to Th3. A 5-day course of systemic corticoids was administrated, with no effect on symptoms.

Etiologic work-up was performed, including coagulation studies (platelet count and coagulation tests (INR/APTT)) and a complete spinal arteriography. No underlying disease could be identified. At two year follow up, he still suffers from a paresis of the lower limbs, but is able to walk with support. He has a kyphosis and a neurogenic bladder dysfunction. The miosis and anhidrosis improved. His hand function recovered slowly to normal. Follow up MRI shows permanent damage to the spinal cord with signs of atrophy.

\section{Case report 2}

A one-and-a-half-year-old boy presented at the emergency department with irritability and crying. The physical examination was normal except for hyperperistalsis. Ultrasound of the abdomen showed mild mesenteric lymphadenitis and spontaneous recovery of ileo-ileal invagination. After two days, the boy returned to the emergency department with an ataxic gait pattern with, frequent fall, right-sided miosis and mild right-sided ptosis. Laboratory examination at admission showed a CRP of $6.6 \mathrm{mg} / \mathrm{l}$ and a white blood cell count of $16.700 / \mathrm{mm}^{3}$. Platelet count and coagulation tests (INR/APTT) were normal. Computed tomography of the brain and cervical spine $(\mathrm{C} 1$ -C4) did not reveal any pathologic findings. Lumbar puncture was performed, showing 9 red blood cells $/ \mathrm{mm}^{3}$ and 36 white blood cells/ $\mathrm{mm}^{3}$ with an elevated protein level of $880 \mathrm{mg} / \mathrm{l}$ and normal lactate and glucose levels. Cultures and serology were negative. A brain and spinal cord MRI was performed two days later, which showed an epidural hematoma extending from C3 to T2 with significant spinal cord compression (Figure 2). There were no intracranial abnormalities.

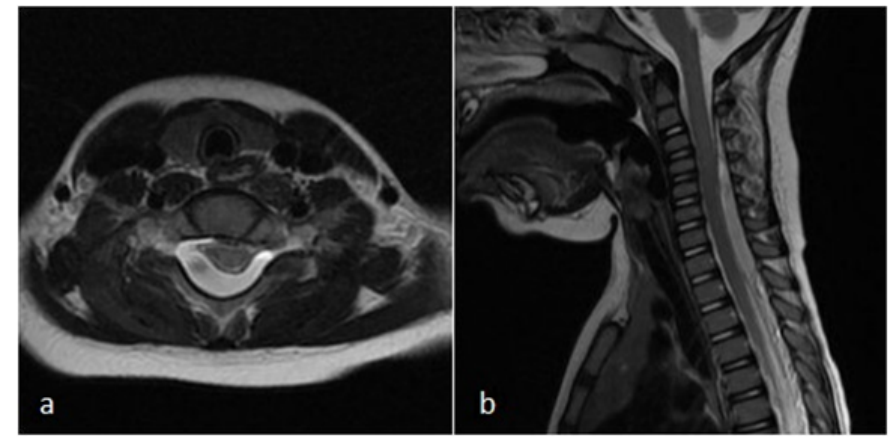

Figure 2. a) Axial T2 MRI showing spinal epidural hematoma at level C6; b) sagittal T2 MRI images of the cervical spinal epidural hematoma extending from level C3 to T3 with spinal cord compression.

Laminectomy with drainage was performed. There was a complete neurologic recovery in the following days.

\section{Discussion}

We describe two young children with a spinal epidural bleeding. Both of them presented initially with a probable viral infection. The first child presented with a respiratory tract infection with nonspecific initial symptoms, including back pain, general weakness and irritability. In retrospect, the back pain seemed to be present several days before admission and was at first attributed to the pneumonia, as was the general weakness and irritability. The second child presented with signs of a mesenteric lymphadenitis. In both children the neurologic focal signs developed later in the course of the disease. This is in line with most pediatric cases described in the literature. The first presenting sign is often back or neck pain, which is present for several hours to days before the onset of the neurologic deficits [5]. In infants the diagnosis is even more challenging with irritability as sole initial symptom [6]. There is a high risk for delay in diagnosis and consequently, delay in treatment.

Besides the motor and sensory deficits, there was also presence of autonomic dysfunction. Our first patient had a partial Horner syndrome with miosis and anhidrosis to the right side. There was a bladder dysfunction. The patient in the second case report presented with right-sided miosis and ptosis. We know that spinal cord lesions in general can lead to a broad range of autonomic abnormalities. This is explained by disruption of connections between higher brain centers and the spinal cord or impairment of the autonomic system itself [7].

Diagnosis is made preferentially by spinal MRI because its capabilities for tissue differentiation [8]. A plain CT scan can be helpful in diagnosing a spinal epidural hematoma, especially in the cervical region. However, the possibility of misdiagnosis remains [8].

No identifiable cause for the bleeding was found in our cases. However, a respiratory tract infection (coughing, infectious state), is described as a predisposing factor in previous cases $[3,4]$ and could possibly explain the vulnerability to bleeding in the first case.

The mainstay of treatment is surgical decompression $[9,10]$ Conservative management can be considered in cases with minimal neurological symptoms and signs of fast resolution [9]. But most often, surgical decompression of the neural structures and removal of the hematoma is necessary [9]. Outcome is mainly determined by the preoperative neurological status $[11,12]$ and the use of anticoagulants [11]. The interval from symptom onset to surgery doesn't seem to be a primary factor in determining prognosis $[11,12]$. However, urgent 
surgical decompression is still recommended in all symptomatic patients [12]. In those with incomplete cord injury this is even of greater importance, since they have the best chances to regain full neurological function [12].

A review of 27 pediatric cases, described by Pecha [10] showed complete neurologic recovery in 14 of 27 (52\%) patients, partial recovery in 12 of 27 (44\%) patients, and death in 1 of 27 (4\%) patients.

In our cases, the second child recovered completely within several days after the laminectomy. In the first child there was a partial (but relatively good) recovery after the first operation. Unfortunately, within 3 days after initial surgical decompression, there was an acute clinical deterioration with development of a rebleed in the same region. Recurrence of SSEH is very rare. There are two types described in the literature: spontaneous or postoperative recurrence [13]. Direct postoperative recurrence is most often associated with the existence of predisposing risk factors (antiplatelet therapy, coagulopathy, vascular malformation), which were absent in our case. In the three pediatric cases of spontaneous recurrent SEH described in the literature, the interval between the first and second SEH varied between 2 months and 6 years.

Despite minimal delay of re-intervention in our first patient, there was permanent damage to the spinal cord with signs of atrophy shown on follow-up MRI. Important neurological deficits remained.

\section{Conclusion}

SSEH is a rare condition, but represents a medical emergency and it should be part of the differential diagnosis of acute back or neck pain and neurologic signs of medullary compression. One has to keep in mind that in most pediatric cases presentation can be atypical and neurologic focal signs can arise hours or days after the onset of back pain.

Awareness for the disease is important for timely diagnosis (by MRI) and treatment, which consists of urgent surgical decompression. Recurrence is very rare, but possible, therefore close clinical follow up after surgery is important. MRI should be repeated in the presence of new or progressive symptoms, so timely intervention can be done.

\section{Acknowledgement}

With support from the University Foundation Belgium.

\section{References}

1. Holtas S, Heiling M, Lonntoft M (1996) Spontaneous spinal epidural hematoma: findings at MR imaging and clinical correlation. Radiology 199: 409-413. [Crossref]

2. Cakir E, Karaarslan G, Usul H, Baykal S, Kuzeyli K, et al. (2004) Clinical course of spontaneous spinal epidural haematoma mimicking Guillain-Barré syndrome in a child: a case report and literature review. Dev Med Child Neurol 46: 838-842. [Crossref]

3. Kreppel D, Antoniadis G, Seeling W (2003) Spinal hematoma: a literature survey with meta-analysis of 613 patients. Neurosurg Rev 26: 1-49. [Crossref]

4. Jumani DB, Littlewood R, Iyer A, Fellows G, Healey A, et al. (2013) Spontaneous spinal epidural haematoma mimicking meningitis in a 2-year-old child - a case report and literature review. Childs Nerv Syst 29: 1795-1798. [Crossref]

5. Patel H, Boaz JC, Phillips JP, Garg BP (1998) Spontaneous spinal epidural hematoma in children. Pediatr Neurol 19: 302-307. [Crossref]

6. Schoonjans AS, De Dooy J, Kenis S, Menovsky T, Verhulst S, et al. (2013) Spontaneous spinal epidural hematoma in infancy: review of the literature and the "seventh" case report. Eur J Paediatr Neurol 17: 537-542. [Crossref]

7. Hou S, Rabchevsky AG (2014) Autonomic consequences of spinal cord injury. Compr Physiol 4: 1419-1453. [Crossref]

8. Shima H, Yasuda M, Nomura M, Mori K, Miyashita K, et al. (2012) A spinal epidural hematoma with symptoms mimicking cerebral stroke. Nagoya J Med Sci 74: 207-210. [Crossref]

9. Groen RJ (2004) Non-operative treatment of spontaneous spinal epidural hematomas a review of the literature and a comparison with operative cases. Acta Neurochir (Wien) 146: 103-110. [Crossref]

10. Pecha MD, Able AC, Barber DB, Willingham AC (1998) Outcome after spontaneous spinal epidural hematoma in children: case report and review of the literature. Arch Phys Med Rehabil 79: 460-463. [Crossref]

11. Bakker NA, Veeger NJ, Vergeer RA, Groen RJ (2015) Prognosis after spinal cord and cauda compression in spontaneous spinal epidural hematomas. Neurology 84: 1894903. [Crossref]

12. Rajz G, Cohen JE, Harnof S, Knoller N, Goren O, et al. (2015) Spontaneous spinal epidural hematoma: the importance of preoperative neurological status and rapid intervention. J Clin Neurosci 22: 123-128. [Crossref]

13. Luo XB, Zhou X, Wang Q, Cai XJ, Luo ZP, et al. (2016) The classification of recurrent spinal epidural hematoma: a review of the literature and a comparison with the cases. Eur Spine J 25 Suppl 1: 224-229. [Crossref]

Copyright: (C2016 Smeets N. This is an open-access article distributed under the terms of the Creative Commons Attribution License, which permits unrestricted use, distribution, and reproduction in any medium, provided the original author and source are credited. 